\title{
Modulation of Central Synapses by Astrocyte-Released ATP and Postsynaptic P2X Receptors
}

\author{
Eric Boué-Grabot ${ }^{1,2}$ and Yuriy Pankratov ${ }^{3,4}$ \\ ${ }^{1}$ Institut des Maladies Neurodégénératives, University Bordeaux, UMR 5293, 33000 Bordeaux, France \\ ${ }^{2}$ Institut des Maladies Neurodégénératives, CNRS, UMR 5293, 33000 Bordeaux, France \\ ${ }^{3}$ School of Life Sciences, University of Warwick, Coventry, UK \\ ${ }^{4}$ School of Life Sciences, Immanuel Kant Baltic Federal University, Kaliningrad, Russia
}

Correspondence should be addressed to Eric Boué-Grabot; eric.boue-grabot@u-bordeaux.fr

Received 24 March 2017; Accepted 10 July 2017; Published 6 August 2017

Academic Editor: Antonio Pinto-Duarte

Copyright ( 2017 Eric Boué-Grabot and Yuriy Pankratov. This is an open access article distributed under the Creative Commons Attribution License, which permits unrestricted use, distribution, and reproduction in any medium, provided the original work is properly cited.

\begin{abstract}
Communication between neuronal and glial cells is important for neural plasticity. P2X receptors are ATP-gated cation channels widely expressed in the brain where they mediate action of extracellular ATP released by neurons and/or glia. Recent data show that postsynaptic P2X receptors underlie slow neuromodulatory actions rather than fast synaptic transmission at brain synapses. Here, we review these findings with a particular focus on the release of ATP by astrocytes and the diversity of postsynaptic P2X-mediated modulation of synaptic strength and plasticity in the CNS.
\end{abstract}

\section{Introduction}

Adenosine 5'-triphosphate (ATP) is arguably one of the most abundant molecules in living cells serving as universal energy "currency." So, it is of no surprise that ATP is also widely utilized as an extracellular signaling molecule [1]. Extracellular ATP acts as an excitatory neurotransmitter in the brain, spinal cord, and peripheral nerve terminals [2-4]. Furthermore, ATP can act as a "gliotransmitter" transferring signals within glial networks or between neurons and glial cells [2-5]. The metabolic breakdown of extracellular ATP by ectonucleotidases is also a source of other nucleotides and adenosine. Adenosine acts as neurotransmitter and neuromodulator in the central nervous system (CNS) through activation of adenosine $G$ protein-coupled receptors that are widely expressed in glia and neurons at both pre- and postsynaptic levels. Adenosine plays important physiological roles in the brain in health and diseases detailed in recent review articles [6-10]. The action of ATP as neuro- or gliotransmitter is mediated by a broad family of purinergic receptors expressed in neurons and glia. P2 receptors are classified into several subtypes of ligand-gated ion channels (P2X1-P2X7 subunits) and eight distinct $\mathrm{G}$ protein-coupled receptors (P2Y) which are both characterized by a variety of distinct properties and a broad range of ATP sensitivities ranging from nanomolar (P2Y receptor) to tenth micromolar (P2X) or millimolar for P2X7 [1, 11-14]. The seven P2X subunits share a unique and simple architecture with two hydrophobic membrane-spanning domains separated by a large extracellular domain and two intracellular termini. They assemble as homo- or heterotrimers to form diverse nonselective cation channels with distinct kinetics and pharmacological properties. All P2X subunits are expressed in neural cells in a heterogeneous manner through the brain regions, cell types, and subcellular compartments [15-17]. Consequently, the subunit composition of $\mathrm{P} 2 \mathrm{X}$ receptors in most of central neurons is far from being characterized. Neuronal P2 receptors are expressed at pre- and postsynaptic loci [18]. Presynaptic P2 receptors play a critical role in the regulation of neurotransmitter release $[10,11]$ by contributing to the intracellular $\mathrm{Ca}^{2+}$ signaling $[11,13]$ by virtue of the high $\mathrm{Ca}^{2+}$ permeability (P2X) and ability to stimulate IP3-dependent $\mathrm{Ca}^{2+}$ release from endoplasmic reticulum (P2Y). These properties can underlie also an important role for postsynaptic P2X 
receptors in the modulation of synaptic activities highlighted relatively recently [12]. In this review, the recent knowledge on the role of postsynaptic $\mathrm{P} 2 \mathrm{X}$ receptors focused on glianeuron interactions is summarized.

\section{Release of ATP by Glial Cells}

An ability of astrocytes to release ATP has been suggested by studies showing the participation of ATP in the propagation of glial $\mathrm{Ca}^{2+}$ waves and the significant contribution of ATP and adenosine to the astroglia-driven modulation of neuronal activity and sleep homeostasis [3, 19-21]. A variety of molecular mechanisms of ATP release from astrocytes have been suggested, including exocytosis and concentration gradient-driven diffusion through large conductance channels such as gap junction hemichannels, anion channels, and dilated P2X7 receptors [3, 5, 21].

In addition to astrocytes, a significant amount of extracellular ATP can be released from microglia, in particular during neuroinflammation [2, 22-24]. Microglia-derived ATP has been reported to activate $\mathrm{P} 2 \mathrm{X}$ receptors in the hippocampal and spinal cord neurons [22-24].

From the early days of research into glial-neuron interaction, a concept of fast vesicular release of chemical transmitters, including ATP, from astrocytes attracted a big attention and was embedded in the popular concept of tripartite synapse [25] which had implied the equal importance of astrocytes for synaptic physiology. Indeed, there is a large body of evidence that the release of ATP from astrocytes may share common mechanisms of vesicular neurotransmitter release such as a dependence on the proton gradient, vesicular transporters, and SNARE proteins and intracellular $\mathrm{Ca}^{2+}$ elevation [20, 26-29]. There are also accumulating reports of physiological roles for SNARE-dependent glial exocytosis $[19,21,28]$. In particular, exocytosis of ATP followed by its conversion to adenosine has been implicated into the regulation of LTP in the the hippocampus and sleep homeostasis in the hypothalamus [21]. The key element of latter works was the development of dnSNARE transgenic mice with inducible inhibition of exocytosis selectively in astrocytes [21].

Yet, the physiological relevance of vesicular release of gliotransmitters is intensively debated $[26,27]$. This debate has been fuelled by an argument that the bulk of evidence supporting the SNARE-dependent release of gliotransmitters was obtained mostly in cell cultures where functional properties of astrocytes can be dramatically altered by various artefacts [27]. Until recently, there were mainly circumstantial evidence of $\mathrm{Ca}^{2+}$-dependent release of ATP from glial cells in brain slice based on the inhibitory action of $\mathrm{P} 2 \mathrm{X}$ receptor antagonists on glia-driven modulation of synaptic transmission and plasticity $[19,30,31]$. A definitive evidence of vesicular release of ATP from astrocytes in situ, based on several experimental approaches, has been demonstrated only recently $[28,32,33]$. These works have demonstrated that quantal release of ATP from cortical astrocytes can be triggered by the cytosolic $\mathrm{Ca}^{2+}$ elevation, attainable in physiological conditions $[28,32,33]$ and can be inhibited by the intracellular perfusion of astrocytes with light chain of tetanus toxin or inhibitors of vesicular nucleotide transporters. The release of ATP was impaired in the transgenic mice expressing dnSNARE protein selectively in astroglial cells $[28,33]$. One should emphasize that this effect could hardly be attributed to the allegedly "leaky" expression [34] of dnSNARE transgene in neurons since there was no deficit in the GABAergic and glutamatergic synaptic transmission $[28,33]$. Also, the lack of neuronal expression of dnSNARE has been recently verified by other labs $[35,36]$. The release of ATP from cortical astrocytes can occur, most likely, from synaptic-like microvesicles and lysosomes [28]. Importantly, these works also provide a direct demonstration of capability of astrocyte-derived ATP to activated P2X receptors in neurons, most likely located at the extrasynaptic sites [28].

The above works also reported a presence of nonvesicular release of ATP from astrocytes which is in agreement with previous data suggesting a participation of connexin hemichannels and/or volume-sensitive channels in glia release of ATP $[3,30,37]$. However, the contribution of nonvesicular mechanisms into the release of ATP from cortical astrocytes at physiological conditions was not large and the dominant role was played by vesicular mechanisms [28]. The major contribution of vesicular mechanisms into the release of ATP has also been reported for brainstem astrocytes in vivo [19]. The notion of the coexistence of vesicular and nonvesicular mechanisms of ATP release and the dominant role of vesicular pathway are in line with previous results obtained in the spinal cord astrocytes [37]. The latter work showed that fast initial astroglial exocytosis of ATP triggered a robust, but slow, secondary release of ATP through the pannexin and connexin hemichannels [37]. One should also note that the data supporting a major role for nonvesicular mechanisms of ATP release in the brain were often reported by works aimed to study the excitotoxicity of ATP after ischemic tissue damage or during neurological disorders [2, 3, 38]. Thus, a consensus view on the mechanism of glial ATP release might be a participation of both exocytosis- and channel-dependent pathways, with the relative contributions former being smaller at physiological conditions but increasing in the context of brain pathologies. It is also feasible that relative contribution of vesicular/nonvesicular mechanisms into gliotransmitter release can be region-specific as many other functions of astrocytes. There is also evidence that the release of ATP from astrocytes can undergo significant agerelated changes [39]. Whatever is a mechanism, the release of ATP represents a powerful pathway of glia-neuron interaction which has already been implicated into a variety of physiological functions, including breathing control [19], slow neuromodulation [30, 31, 40], and modulation of sleep homeostasis [21] (after conversion into adenosine). Yet, the most intriguing and frequently overlooked role for gliaderived ATP is the regulation of synaptic strength, which will be discussed in detail below.

\section{Neuromodulatory Action of Postsynaptic P2X Receptors in the CNS}

ATP showed to be released by exocytosis from the nerve terminal and acts as a fast synaptic neurotransmitter in the 
nervous system though activation of postsynaptic P2X receptors [41-46]. If there is robust evidence of fast transmission mediated by ATP and P2X receptors in the peripheral nervous system such as sympathetic neurons, at neuromuscular junction or in myenteric nervous system, such a form of ATP-mediated fast synaptic transmission is more rarely observed in the CNS (see for review [11]). Following the first evidence for fast excitatory ATP postsynaptic currents recorded in the medial habenula $[41,42]$, fast ATP synaptic transmission mediated by activation of postsynaptic $\mathrm{P} 2 \mathrm{X}$ receptors was also reported in several other brain regions such as the locus coeruleus, the hippocampus, the hypothalamus, and the cortex as well as in the spinal cord [5, 43, 44, 46, 47]. However, evoked P2X-mediated postsynaptic currents required generally strong electrical stimulation of afferent fibers and are of weak amplitude [32, 45]. To date, there is no example of action potential firing induced by synaptic release of ATP and activation of P2X receptors in the CNS. In addition, rare spontaneous or miniature P2X-mediated excitatory postsynaptic currents were recorded at central synapses conveying the idea that ATP and postsynaptic P2X receptors do not act primarily as fast excitatory neurotransmitter at brain synapses [12] and, thus, despite the detection of several P2X subunits at the postsynaptic sites of glutamatergic synapses [15]. Several studies suggested that postsynaptic P2X receptors modulate fast synaptic responses mediated by other fast neurotransmitters (see for review $[12,48]$ ). Recent works have showed that ATP released by glial cells and/or by the presynaptic terminals acting on postsynaptic P2X receptors have various and profound effects on synaptic efficacy and plasticity at both the excitatory and inhibitory synapses in several brain areas [28, 30-32, 40,49]. There is also evidence of abundant expression of various P2X receptor subtypes at presynaptic loci [15] and presynaptic effects of $\mathrm{P} 2 \mathrm{X}$ and P2Y receptors on the release of glutamate, GABA, and other neurotransmitters in different brain regions and the spinal cord $[1,10,11,15,50-52]$. These results gave rise to the hypothesis that the main role for ATP and P2Xmediated signaling in CNS is neuromodulation rather than fast neurotransmission [12, 52].

Although the presynaptic action of the $\mathrm{P} 2$ receptors on the release of various neurotransmitters have been studied for last two decades (see for review $[10,11]$ ), the notion of physiological importance of the postsynaptic purinergic modulation has been highlighted relatively recently [12]. Several arguments were already in favor of the modulatory action of ATP released from nerve terminals and postsynaptic P2X receptors. The first is that no brain synapses to date used solely ATP as excitatory neurotransmitter but ATP is frequently coreleased from the same terminals with other transmitters such as glutamate or $\gamma$-amino-butyric acid (GABA) [32, 43, 44, 47, 53]. Consistent with this idea, electron microscopy indicated that P2X2, P2X4, and $\mathrm{P} 2 \mathrm{X} 6$, the predominant fast purinoceptors expressed in the majority of brain neurons, are postsynaptically located and mainly localized at the edge of the postsynaptic density synapses in the peri- and extrasynaptic space [16] at least at the basal levels of neuronal activity. In contrast,
AMPAR or $\mathrm{GABA}_{\mathrm{A}} \mathrm{R}$ which mediate, respectively, the rapid excitatory and inhibitory synaptic transmission are concentrated at the synapses facing the site of neurotransmitter release from the presynaptic terminal $[54,55]$. Single-molecule imaging methods allow now the visualization of the lateral movement of receptors in the postsynaptic membrane. They have revealed that AMPAR and $\mathrm{GABA}_{\mathrm{A}} \mathrm{R}$ permanently exchange between synaptic and extrasynaptic locations and alternate between periods of lateral mobility at extrasynaptic sites, and periods of immobility at synapses [56]. In contrast, single particle tracking of $\mathrm{P} 2 \mathrm{X} 2$ or $\mathrm{P} 2 \mathrm{X} 4$ receptors has shown that they are all very mobile and that their diffusion can be facilitated upon their activation by ATP [57]. In transfected neurons, P2X2 receptors moved within the membrane of dendrites and at extrasynaptic sites. In addition, these data showed that $\mathrm{P} 2 \mathrm{X} 2$ receptors rarely enter into the glutamatergic synapse suggesting that $\mathrm{P} 2 \mathrm{X}$ receptors are excluded from the postsynaptic density synapses reinforcing the electron microscopy data [58].

The lack of detectable P2X-mediated synaptic current is also consistent with the fact that the surface trafficking of $\mathrm{P} 2 \mathrm{X} 4$ receptors is highly regulated. $\mathrm{P} 2 \mathrm{X} 4$ receptors are constitutively internalized by dynamin-dependent endocytosis $[59,60]$ through interaction between the adaptor protein AP2 and a novel noncanonical endocytosis motif present exclusively in P2X4 subtype $[59,61,62]$. As a result, P2X4-containing receptors are retained predominantly in intracellular pools, limiting their putative implication in ATP signaling originating from either neuron or glia in normal conditions. However, intracellular pools of P2X4 receptors that are resistant to lysosomal degradation can be rapidly trafficked to the surface membrane increasing the surface number of functional P2X4 receptors [63]. Apart from P2X2, P2X4, and P2X6 subtypes, the presence of other subtypes, such as P2X1 or P2X3, in the central synapses has been suggested based both of the electrophysiological/pharmacological data and colocalisation with synaptic markers $[15,44,45]$; the presence of heteromeric P2X1/4 and P2X2/ 3 also cannot be excluded. Since P2X1 and P2X3 receptors are prone to the fast desensitisation, internalisation, and lateral mobility $[1,64]$, the subtype composition and overall density of $\mathrm{P} 2 \mathrm{X}$ receptors at the given synapse may undergo substantial variations, very likely in the activity-dependent manner. Interestingly, a recent study of $\mathrm{P} 2 \mathrm{X}$ receptormediated signaling in individual cortical synapses suggested that ATP released either from synaptic terminals or from astrocytes can upregulate the trafficking of postsynaptic P2X receptors via different $\mathrm{Ca}^{2+}$-dependent mechanisms [32].

This is likely to be important because an upregulation of surface P2X receptors (in particular P2X4 subtype) was observed in various pathophysiological contexts such as ischemia, chronic pain [23], or neurodegenerative diseases such as amyotrophic lateral sclerosis (ALS) or Alzheimer's disease (AD) [38, 65-67]. So, excessive release of ATP from various sources, including astrocytes and microglia, can confer a particular importance of the neuromodulatory action of $\mathrm{P} 2 \mathrm{X}$ receptors in pathological conditions. 


\section{Modulation of Glutamatergic Excitatory Synapse by P2X Receptors}

NMDAR and AMPAR have critical roles in excitatory synaptic transmission and plasticity in the CNS [68]. Synaptic strength is thought to be determined in part through the activity-dependent insertion and removal of synaptic AMPAR which lead to long-term potentiation (LTP) and long-term depression (LTD), that are widely recognized to underlie cognitive functions such as learning and memory [68-70]. AMPARs are tetrameric complexes composed of GluA1-A4 subunits [71]. The lateral diffusion and the intracellular trafficking (endocytosis/exocytosis) are regulated by subunit-specific AMPAR-interacting proteins as well as by various posttranslational modifications on their intracellular domains $[72,73]$. The bidirectional fast changes in the number of synaptic AMPARs are mainly triggered by activation of NMDAR or mGluR during synaptic plasticity.

4.1. Direct Modulation of AMPAR and Glutamatergic Synapse Efficacy by Glial ATP. Two noteworthy studies showed that direct activation of postsynaptic P2X receptors by endogenous ATP plays also a crucial role in glutamatergic synaptic transmission [30,40]. These two works provide strong evidence that postsynaptic P2X receptors activated by ATP released from astrocytes resulted in enduring changes of glutamatergic synaptic efficacy in distinct brain areas. The first work showed in the paraventricular nucleus of the hypothalamus, a long lasting increase in the amplitude of AMPAR-mediated mEPSC in response to brief application of noradrenaline (NA) that requires the release of ATP from glial cells [30]. It has been demonstrated that NA acted on astrocytic $\alpha 1$-adrenoceptors and triggered the release of ATP by astrocytes. In addition, the pharmacological blockade by fluoroacetate (FAC) of the glial metabolism or physiological decrease by dehydration of the astrocytic coverage of the synapses onto magnocellular neurons in the hypothalamic neurosecretory system suppressed the increase in synaptic strength. The increase of AMPAR-mediated mEPSC is mediated by $\mathrm{P} 2 \mathrm{X}$ receptors' activation and calcium influx and phosphatidylinositol 3-kinase (PI3K) resulting likely in the insertion of new AMPAR at the synapse. The effect of NA on the AMPAR-mediated mEPSCs was mimicked by ATP or benzoyl ATP, a more stable analogue of ATP, and blocked in the presence of brilliant blue $\mathrm{G}$ (BBG) in favor of the involvement of $\mathrm{P} 2 \mathrm{X} 7$ receptors [30]. P2X7 subunit is possibly expressed in the magnocellular neurons of the hypothalamus with several other subunits [17]; but P2X7 is rarely expressed in neurons suggesting that this mechanism could be restricted to specific neuronal populations. It was showed later that P2X2 and P2X 5 formed a heteromeric P2X receptor with an apparent affinity for ATP similar to P2X2 $(\sim 10 \mu \mathrm{M})$ and P2X7-like functional properties [74]. This suggests that such form of plasticity could be mediated by more classical neuronal P2X receptors and consequently more broadly expressed in the brain (Figure 1).

The second work showed that activation of postsynaptic P2X (likely P2X2) receptors by NA-dependent glial release of endogenous ATP changed the glutamatergic synapses efficacy in the hippocampus but in an opposite way [40]. Brief application of ATP or NA-induced glial ATP release caused an enduring and slow decrease of AMPAR-mediated mEPSCs in hippocampal cultures. The effects of both ATP and NA were blocked by PPADS, a P2X antagonist, and by FAC that impairs astrocyte function. The same observation was reproduced on hippocampal brain slices where ATP and NA caused a depression of field excitatory postsynaptic potentials (fEPSPs) recorded in CA1 region that was also blocked by PPADS or FAC. By complementary approaches, including recordings and biochemistry from heterologous Xenopus oocytes expressing mammalian P2X2 or P2X4 with AMPAR, and high-resolution imaging from hippocampal neurons, Pougnet and colleagues demonstrated that activation of $\mathrm{P} 2 \mathrm{X} 2$ or P2X4 receptors and calcium influx though the opening of P2X channels triggered dynamin-dependent internalization of GluA1 or GluA1/A2 AMPARs leading to reduced surface AMPARs in dendrites and at synapses [40].

Although no fast ATP synaptic transmission was detected in both brain structures, these studies showed that ATP released by astrocytes increased the efficacy of glutamatergic synaptic transmission in paraventricular nucleus and depressed synaptic efficacy in the CA1 region of the hippocampus $[30,40]$. This raised the possibility that astrocytederived ATP and P2X receptors may bidirectionally change the glutamatergic synapse efficacy in the brain.

Gordon and colleagues have also established that the ATP signaling mechanism is engaged during an afferent activity [31]. The activity-dependent release of NA or glutamate by nerve terminals activates astrocytic mGluR or adrenoceptors (Figure 1). The subsequent increase of intracellular calcium in astrocyte leads to the release of ATP by astrocytes and consequently activation of postsynaptic $\mathrm{P} 2 \mathrm{X}$ receptors of a large group of synapses covered by the astrocyte. Astrocytic activation may spread within astrocytes resulting in multiplicative scaling of all synapses in MCNs. Thus, although not contributing to the synaptic transmission, astrocyte-derived ATP signaling is likely exerting strong neuromodulation during physiological neuronal activation [31]. In addition, recent studies $[75,76]$ showed that startle and arousal responses in vivo are followed by NA-induced $\mathrm{Ca}^{2+}$ signaling in cortical astrocytes raising also the possibility that $\mathrm{P} 2 \mathrm{X}$ receptors may contribute to the regulation of these behaviours [29].

The P2X-induced bidirectional changes in the efficacy of glutamatergic transmission induce insertion and removal of AMPAR at the synapses [30, 40]. P2X-mediated insertion of AMPAR in MCNs is linked to PIK3 kinase [30] that is also crucial for NMDA-dependent AMPAR insertion and LTP [69] suggesting that $\mathrm{P} 2 \mathrm{X}$ receptors may serve as a "surrogate" for NMDAR [30]. In the hippocampus, P2X- and NMDAinduced depression in the CA1 region were additive, suggesting that both receptors are involved in distinct mechanisms leading to the removal of AMPAR from the synapses [40]. This assumption was confirmed recently by a work showing that P2X-mediated internalization of AMPAR is associated to the phosphorylation level of two calcium/calmodulindependent protein kinase II (CaMKII) phosphorylation sites S567 and S831 located in the cytoplasmic Loop1 and Cterminal tail of GluA1 subunits [49]. This work revealed also 


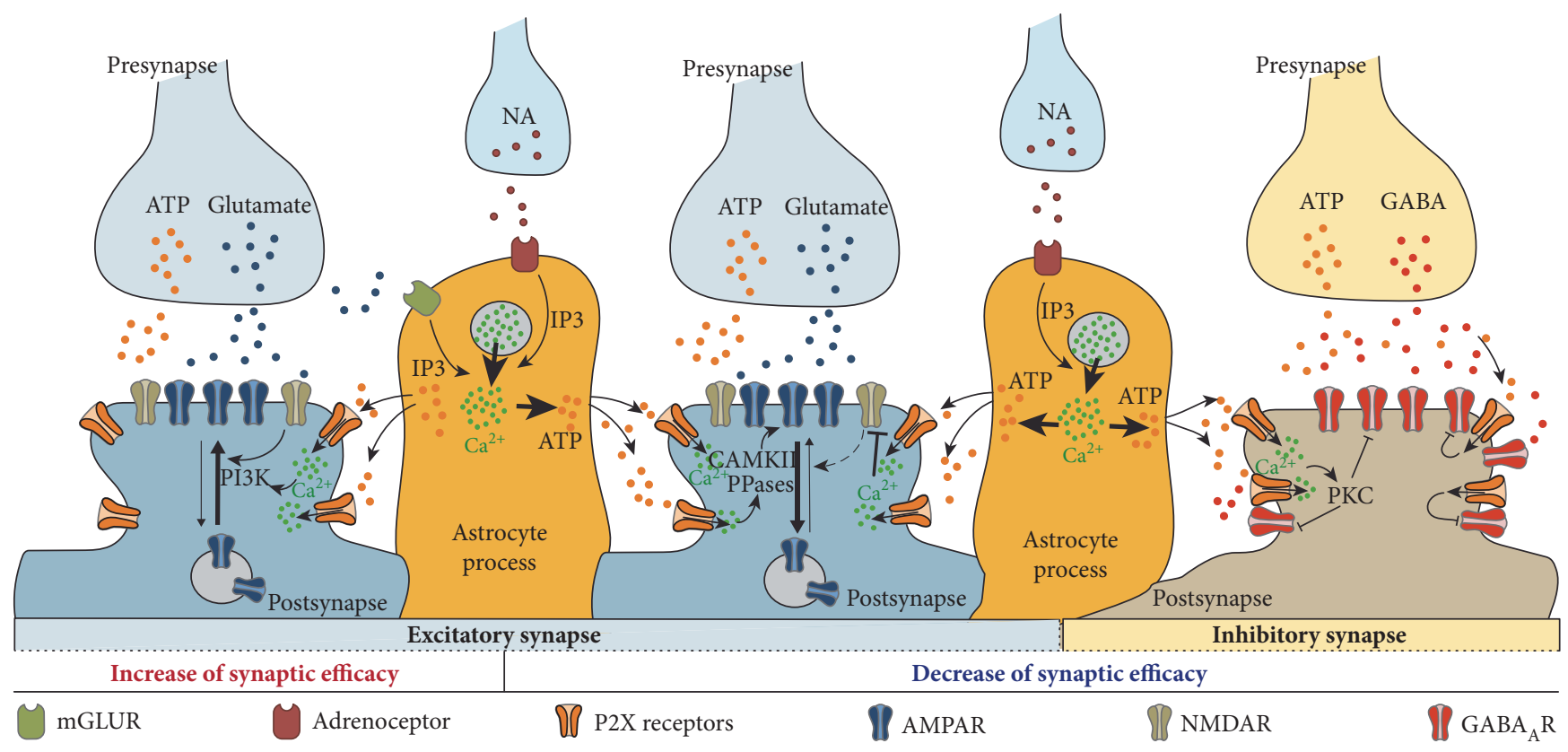

FIgURE 1: Summary of the postsynaptic P2X-mediated modulation of synaptic strength and plasticity following the release of ATP by astrocytes or neurons. Glutamate and noradrenaline acting on astrocytes cause the release of glial ATP. Activation of postsynaptic P2X receptors at glutamatergic synapses (blue) can trigger either a PIK3-dependent insertion of AMPAR leading to the increase of synaptic strength and synapse scaling (left) or a CaMKII-dependent internalization of AMPAR leading to a P2X-dependent synaptic depression (middle). P2X receptors can also cause an alteration of glutamatergic synapse plasticity by inhibiting NMDA function by interfering on PSD-95/NMDAR complex. At inhibitory synapses (brown), glial or neuronal ATP decreases GABAergic synapse efficacy by direct or indirect alteration of $\mathrm{GABA}_{\mathrm{A}}$ (right).

that P2X-mediated AMPAR inhibition is dependent on the subunit composition of AMPAR. GluA1 or GluA1/A2 are regulated by $\mathrm{P} 2 \mathrm{X}$ activation while GluA3 homomers are insensitive and their presence in heteromers alters P2Xmediated inhibition. In addition, P2X-mediated depression in CA1 P2X-induced depression in hippocampal slices produces a dephosphorylation of the GluA1 subunit at S567, contrary to NMDAR-mediated LTD [49] (Figure 1).

Opposite effects of P2X-mediated changes of glutamatergic synapses' efficacy were both triggered by the $\mathrm{Ca}^{2}$ influx through the opening of $\mathrm{P} 2 \mathrm{X}$ channels that activate distinct intracellular pathways regulating AMPAR trafficking. Does postsynaptic P2X receptors may bidirectionally change the efficacy of a synapse depending on astrocytic release of ATP, the dynamic of the $\mathrm{Ca}^{2+}$ influx though P2X receptors, or the nature of AMPAR? Such possibility will be of interest but requires additional studies since opposite effects were observed in distinct neuronal population recruiting very likely distinct P2X receptors.

4.2. Modulation of NMDAR-Dependent Plasticity. The NMDAR-dependent LTP in hippocampal CA1 neurons was shown to be modulated by P2X manipulation. Pharmacological blockade of $\mathrm{P} 2 \mathrm{X}$ facilitated the induction of NMDA-dependent LTP [46, 77] at lower frequencies of action potential firing in Schaffer collateral axons suggesting that $\mathrm{P} 2 \mathrm{X}$ receptor activation reduced NMDA-dependent LTP at low frequency stimulation [46, 77]. In contrast, a study using P2X4 knockout mice revealed that LTP in CA1 neurons was slightly reduced compared to that in wild-type mice [78]. In addition, ivermectin, a positive modulator of P2X4 function or trafficking $[79,80]$, enhanced LTP in the wild-type but not in P2X4 knock-out mice indicating a facilitatory action of P2X4 for hippocampal LTP [78]. No change of the fast excitatory synaptic transmission between P2X4 knockout and wild-type mice confirmed the absence of contribution of P2X4 in synaptic transmission, and the results suggested that P2X4 receptors regulated NR2B content within synaptic NMDAR [81].

Although controversial, altogether, these data argue in favor of the neuromodulatory action of ATP signaling rather than in fast synaptic transmission but also indicate that function of P2X receptors on NMDAR-dependent plasticity was far to be understood. Recently, a study dissected the role of synaptic and glial release of ATP in individual cortical synapses [32]. This work provided the direct evidence that ATP released from the individual excitatory synapses and/or glial cells can activate P2X receptors in the neocortical neurons. Moreover, the release of ATP from glial astrocytes turned out to trigger a recruitment of $\mathrm{P} 2 \mathrm{X}$ receptors into synapses via $\mathrm{Ca}^{2+}$-dependent mechanism. Dependence of P2X recruitment to synapses on glial activity may explain why, despite evidence of widespread expression of P2X receptors in central neurons, the purinergic components of fast EPSCs are rarely observed and often require stronger stimulation to evoke $[12,45]$.

So, glial and neuronal release of ATP can converge to activation of postsynaptic P2X which, in turn, can cause 
a significant decrease in the synaptic currents mediated by NMDAR (Figure 1). This effect was underlined by the $\mathrm{Ca}^{2+}$-dependent dephosphorylation and interactions between NMDARs and calcineurin within PSD-95 protein complex [32, 82]. P2X-mediated downregulation of NMDA receptors was inhibited in the mutant mice, lacking PSD95/Dlg4 domain (PSD-95 mutants) and the P2X4 knockout mice [32]. As one might expect, the downregulation of NMDAR-mediated synaptic signaling had a strong impact on long-term synaptic plasticity. Impairment of purinergic modulation of NMDAR in the PSD-95 mutant mice led to substantial upregulation of the long-term synaptic plasticity in the neocortex which is manifested in the decrease in the threshold of LTP induction and increase in the net magnitude of LTP. These results are in line with the previous observation of facilitation of LTP induction in CA1 neurons after the inhibition of P2X receptors [46] and previously reported increase in the extent of LTP in PSD-95 mutant mice [82]. One should note that purinergic downregulation of NMDARs was not mediated solely by P2X4; contribution of other $\mathrm{P} 2 \mathrm{X}$ subtypes was suggested by pharmacological data $[32,46]$. The P2Y receptors could bring some, albeit moderate, contribution in the downregulation of NMDARs since this effect was significantly reduced after the removal of extracellular $\mathrm{Ca}^{2+}$ and could be mimicked to a large extent by application $\alpha, \beta$-meATP-a selective P2X agonist [32, 43].

Interestingly, attenuation of purinergic modulation of NMDARs in P2X4 KO mice also shifted the threshold of LTP induction towards weaker stimuli but reduced the net LTP amplitude. In general, such reduction of the LTP amplitude in the neocortex agrees with previously reported decrease of LTP magnitude in the CA1 neurons of P2X4 KO mice [78]. The diversity of effects exerted by P2X receptors on the LTP suggests an involvement, in addition to inactivation of NMDARs, another molecular cascades such as the abovementioned bidirectional modulation of activity and trafficking of AMPARs and the downregulation of GABA receptors (see below).

Combined, these findings suggest an important role for the interaction between postsynaptic P2X and NMDARs in the regulation of synaptic strength. Coupling of NMDA receptors to PSD-95 and multiprotein complex is essential for the bidirectional modulation of synaptic strength and, therefore, for proper function of synaptic networks underlying learning and memory [82]. It has been shown previously that overenhancement of LTP in the PSD-95 KO mutants is accompanied by severe impairment of different forms of learning [82, 83]. It becomes evident now that this important pathway of control of NMDA receptor activity can be exploited by postsynaptic P2X receptors activated by gliaderived ATP. Such "premoderation" of plasticity of excitatory synapses can help to avoid "unwanted" or "excessive" LTP, that is, reduce number of errors. This mechanism can also increase a dynamic range of potentiation by presetting the lower baseline level of the synaptic activity. The P2Xmediated endocytosis of AMPAR [12, 40] can also contribute to this mechanism.

\section{Modulation of GABAergic Inhibitory Synapse}

The modulation of GABAergic system by P2X receptors was first observed by the action of presynaptic P2X receptors located on GABAergic terminals in the retina, spinal cord, or in midbrain where they regulate the release of GABA [50, 84, 85]. Direct modulation by postsynaptic P2X receptors of GABAergic inhibitory synaptic transmission was first identified in the ventromedial nucleus of the hypothalamus $[60,86]$. Blocking the constitutive endocytosis of P2X4 receptors expressed in steroidogenic factor 1 (SF-1) positive neurons increased responses evoked by exogenous application of ATP but did not reveal fast ATP synaptic transmission. However, blocking P2X4 endocytosis depressed inhibitory GABA-mediated postsynaptic currents and consequently enhanced neuronal excitability of SF-1 neurons. GABA current inhibition was independent of ion flow through the $\mathrm{P} 2 \mathrm{X}$ receptors and dependent on a direct interaction between P2X4 and $\mathrm{GABA}_{\mathrm{A}}$ Rs [60]. In addition, disruption of the physical coupling between $\mathrm{P} 2 \mathrm{X} 4$ and $\mathrm{GABA}_{\mathrm{A}}$ receptors using competitive peptides corresponding to the interaction domain identified in the C-terminal tail of P2X4 abolished $\mathrm{P} 2 \mathrm{X} 4$ receptor-mediated GABAergic depression in SF-1 GFP-positive neurons. Molecular and functional crossinhibition was also observed between $\mathrm{P} 2 \mathrm{X} 2$ or $\mathrm{P} 2 \mathrm{X} 3$ and nicotinic, $\mathrm{GABA}_{\mathrm{A}}$ and 5-HT3 receptors in different native or recombinant cells [87-93]. Therefore, we should consider the possibility that $\mathrm{P} 2 \mathrm{X}$ receptors, although almost silent during fast transmission, may be activated by ambient ATP and exert direct action on various other neurotransmitter receptors at central synapses (Figure 1).

Indeed, a recent study demonstrated that ATP released from cortical astrocytes modulated phasic and tonic GABAergic inhibition [28]. In this case, the downregulation of $\mathrm{GABA}_{\mathrm{A}} \mathrm{R}$ was mediated by the calcium entry through the opening of P2X receptors. The downregulation of both tonic and phasic GABAergic transmission relied on the phosphorylation by $\mathrm{PKC}$ and was significantly reduced in the P2X4 KO and dnSNARE mice. Since phasic and tonic GABA conductances can influence membrane depolarization, one may expect that the downregulation of GABA receptors by astrocyte-derived ATP can be implicated in the glial modulation of the long-term potentiation. Indeed, glial exocytosis of ATP has been recently reported to facilitate the induction of LTP in neocortical neurons [33, 94]. This effect is dependent on the activity of P2X receptors $[33,94]$ and can be mimicked by attenuation of GABAergic inhibition by $\mathrm{GABA}_{\mathrm{A}} \mathrm{R}$ antagonists [33]. Most likely, P2Xmediated attenuation of GABAergic inhibition facilitates LTP induction via increasing the depolarization of postsynaptic neurons and, therefore, alleviating the $\mathrm{Mg}^{2+}$ block of NMDA receptors (Figure 1).

\section{Concluding Remarks}

In the ongoing debate on physiological significance of purinergic gliotransmission, the leading role is usually assigned to adenosine receptors which can modulate synaptic transmission and plasticity via presynaptic and postsynaptic 
mechanisms $[2,3,20,21,26,95]$. In the context of glianeuron communications, ATP is often considered merely as a precursor of adenosine $[3,20,26,96]$. However, the recent data discussed in this review highlighted new roles for ATP as a gliotransmitter and importance of $\mathrm{P} 2 \mathrm{X}$ receptors for the modulation of synaptic transmission at postsynaptic sites. Furthermore, some of these data have revealed the dynamic cooperation between synaptic and glial release of ATP in the regulation of synaptic strength. Importantly, ATP released from astrocytes can facilitate a recruitment of P2X receptors into excitatory synapses by $\mathrm{Ca}^{2+}$-dependent mechanism [32] thereby acting upstream of synaptic purinergic signaling. This may underlie a particular physiological relevance of glial release of ATP.

Taken together, the recent data obtained (by different groups) in the different brain regions and physiological/pathological context suggest the existence of complex pathways of glia-derived purinergic regulation of synaptic transmission at postsynaptic loci. These pathways, which include the bidirectional modulation of AMPAR-mediated synaptic transmission, inactivation of NMDAR, and downregulation of GABAergic inhibition, rely on different intracellular signaling cascades. So, the prevalence of one or another pathway will be affected by the level expression of specific proteins (i.e., P2X of different subtypes, PKC, PI3K, CaMKII, and calcineurin) in the particular neuron of particular brain region. At the same time, the release of ATP from astrocytes can be activated virtually by any source of cytosolic $\mathrm{Ca}^{2+}$ elevation $[28,30,32,33,40]$, which reaches certain threshold. Hence, the purinergic gliotransmission may have a different outcome on synaptic plasticity (potentiation versus depression) depending on the physiological context and activity of local networks.

There is growing recognition of the importance of neuronal-glial networks for coordination of metabolism and information processing in the brain, both in health and disease [20, 26, 96-98]. The capability of purinoreceptors, activated by glia-derived ATP, to trigger various $\mathrm{Ca}^{2+}$-dependent intracellular cascades, can make them very important for such coordination. As discussed above, purinergic astroglial modulation of synaptic transmission has many peculiar features, such as slower time-scale, the dependence on the activity of surrounding synapses, and ability to change the balance between excitatory and inhibitory synaptic inputs. These features can underlie an importance of astroglial release of ATP for heterosynaptic metaplasticity [96].

One could argue that coordination of activity over large astroglia syncytium might compromise the synapse specificity of glia-neuron communication, and therefore, the modulatory action of gliotransmitters, in particular ATP, would lack a selectivity needed for Hebbian plasticity. However, synaptic plasticity and memory require cooperation between many cellular and molecular mechanisms within neuronal ensembles, in particular for storage and retrieval of memory $[99,100]$. There is growing consensus that memory and learning rely on distributed spatial and temporal representations of events overlaid in a distributed manner within a common neuronal circuit [100]. So, it is possible that glia-derived "preconditioning" of excitatory and inhibitory synapses within neuronal ensemble, for example, via P2X receptor-linked mechanisms discussed above, can affect the possibility of associations between stimuli/events. Also, there is emerging evidence supporting the physiological importance of fast local $\mathrm{Ca}^{2+}$ events in the astrocytic microdomains [101]. Potentially, astrocytes are capable of localized interaction with individual synapses. The ability of hippocampal and cortical astrocytes to generate $\mathrm{Ca}^{2+}$ elevations in response to activation of single synapse or single axon has been reported $[32,102]$. The activity of astrocytes can also be input-specific [103]. Thus, it is conceivable that local $\mathrm{Ca}^{2+}$ transients in tiny astrocytic processes can trigger spatially constrained release of gliotransmitters, for example, ATP, and selectively affect the activity of an individual synapse. A putative physiological implication of such local glia-neuron communications is yet to be investigated.

Interestingly, several works have shown that astroglial $\alpha 1$-adrenoreceptors can efficiently activate the release of ATP from astrocytes. Recent data have implicated the adrenergic signaling in responsiveness of astrocyte networks to the behavioural state and sensory inputs $[75,76]$. On the other hand, adrenergic neurons of locus coeruleus, the main source of noradrenaline release in the brain cortex, can undergo massive loss during neurodegenerative diseases [104]. Also, the release of ATP from astrocytes, including the one triggered by $\alpha 1$-adrenoreceptors, can decline with ageing [39]. Thus, astroglial release of ATP by astrocytes as well as by microglia and purinergic modulation of synaptic transmission may be of particular importance for the brain metaplasticity induced by experience, environmental factors, ageing, or neurodegenerative disease $[6,96,105]$. Conversely, massive release of ATP occurs also from damaged or dying cells after brain ischemia or trauma or during neurodegenerative diseases $[1,10,38,98]$ and is often correlated to increased expression of P2X receptors [1]. Exacerbating ATP P2X signaling may also have profound effect on brain synaptic activity or plasticity. These very interesting and timely topics are yet to be explored.

To conclude, recent results strongly support the physiological importance of P2X receptors and astroglial release of ATP. The purinoreceptor-mediated communication between astrocytes and neurons is essential for the regulation of synaptic strength and the modulation of synaptic plasticity.

\section{Conflicts of Interest}

The authors declare that they have no competing interests.

\section{Acknowledgments}

This work was supported (Eric Boué-Grabot) by CNRS, Université de Bordeaux, ARSLA-FRC, and LabEx BRAIN ANR-10-LABX-43.

\section{References}

[1] G. Burnstock, "Physiology and pathophysiology of purinergic neurotransmission,” Physiological Reviews, vol. 87, no. 2, pp. 659-797, 2007. 
[2] A. M. Butt, "ATP: a ubiquitous gliotransmitter integrating neuron-glial networks," Seminars in Cell \& Developmental Biology, vol. 22, no. 2, pp. 205-213, 2011.

[3] R. D. Fields and G. Burnstock, "Purinergic signalling in neuron-glia interactions," Nature Reviews Neuroscience, vol. 7, no. 6, pp. 423-436, 2006.

[4] R. D. Fields and B. Stevens, "ATP: an extracellular signaling molecule between neurons and glia," Trends in Neurosciences, vol. 23, no. 12, pp. 625-633, 2000.

[5] U. Lalo, A. Verkhratsky, and Y. Pankratov, "Ionotropic ATP receptors in neuronal-glial communication," Seminars in Cell \& Developmental Biology, vol. 22, no. 2, pp. 220-228, 2011.

[6] K. Harada, T. Kamiya, and T. Tsuboi, "Gliotransmitter release from astrocytes: functional, developmental, and pathological implications in the brain," Frontiers in Neuroscience, vol. 9, p. 499, 2016.

[7] A. M. Sebastiao and J. A. Ribeiro, "Adenosine receptors and the central nervous system," Handbook of Experimental Pharmacology, vol. 193, pp. 471-534, 2009.

[8] P. Popoli and R. Pepponi, "Potential therapeutic relevance of adenosine A2B and A2A receptors in the central nervous system," CNS \& Neurological Disorders Drug Targets, vol. 11, no. 6, pp. 664-674, 2012.

[9] S. Paul, P. H. Elsinga, K. Ishiwata, R. A. Dierckx, and A. Waardevan, "Adenosine $\mathrm{A}(1)$ receptors in the central nervous system: their functions in health and disease, and possible elucidation by PET imaging," Current Medicinal Chemistry, vol. 18, no. 31, pp. 4820-4835, 2011.

[10] R. J. Rodrigues, A. R. Tome, and R. A. Cunha, "ATP as a multi-target danger signal in the brain," Frontiers in Neuroscience, vol. 9, p. 148, 2015.

[11] M. P. Abbracchio, G. Burnstock, A. Verkhratsky, and H. Zimmermann, "Purinergic signalling in the nervous system: an overview," Trends in Neurosciences, vol. 32, no. 1, pp. 19-29, 2009.

[12] B. S. Khakh and R. A. North, "Neuromodulation by extracellular ATP and P2X receptors in the CNS," Neuron, vol. 76, no. 1, pp. 51-69, 2012.

[13] Y. Pankratov and U. Lalo, "Calcium permeability of ligandgated $\mathrm{Ca}^{2+}$ channels," European Journal of Pharmacology, vol. 739, pp. 60-73, 2014.

[14] G. A. Weisman, L. T. Woods, L. Erb, and C. I. Seye, "P2Y receptors in the mammalian nervous system: pharmacology, ligands and therapeutic potential," CNS \& Neurological Disorders Drug Targets, vol. 11, no. 6, pp. 722-738, 2012.

[15] R. J. Rodrigues, T. Almeida, P. J. Richardson, C. R. Oliveira, and R. A. Cunha, "Dual presynaptic control by ATP of glutamate release via facilitatory $\mathrm{P} 2 \mathrm{X} 1, \mathrm{P} 2 \mathrm{X} 2 / 3$, and $\mathrm{P} 2 \mathrm{X} 3$ and inhibitory $\mathrm{P} 2 \mathrm{Y} 1, \mathrm{P} 2 \mathrm{Y} 2$, and/or $\mathrm{P} 2 \mathrm{Y} 4$ receptors in the rat hippocampus," The Journal of Neuroscience, vol. 25, no. 27, pp. 6286-6295, 2005.

[16] M. E. Rubio and F. Soto, "Distinct localization of P2X receptors at excitatory postsynaptic specializations," The Journal of Neuroscience, vol. 21, no. 2, pp. 641-653, 2001.

[17] V. Vavra, A. Bhattacharya, and H. Zemkova, "Facilitation of glutamate and GABA release by P2X receptor activation in supraoptic neurons from freshly isolated rat brain slices," Neuroscience, vol. 188, pp. 1-12, 2011.

[18] B. S. Khakh and R. A. North, "P2X receptors as cell-surface ATP sensors in health and disease," Nature, vol. 442, no. 7102 , pp. 527-532, 2006.
[19] A. V. Gourine, V. Kasymov, N. Marina et al., "Astrocytes control breathing through pH-dependent release of ATP," Science, vol. 329, no. 5991, pp. 571-575, 2010.

[20] M. M. Halassa, T. Fellin, and P. G. Haydon, "The tripartite synapse: roles for gliotransmission in health and disease," Trends in Molecular Medicine, vol. 13, no. 2, pp. 54-63, 2007.

[21] M. M. Halassa, C. Florian, T. Fellin et al., "Astrocytic modulation of sleep homeostasis and cognitive consequences of sleep loss," Neuron, vol. 61, no. 2, pp. 213-219, 2009.

[22] E. Beamer, F. Goloncser, G. Horvath et al., "Purinergic mechanisms in neuroinflammation: an update from molecules to behavior," Neuropharmacology, vol. 104, pp. 94-104, 2016.

[23] S. Beggs, T. Trang, and M. W. Salter, "P2X4R+ microglia drive neuropathic pain," Nature Neuroscience, vol. 15, no. 8, pp. 1068-1073, 2012.

[24] J. George, R. A. Cunha, C. Mulle, and T. Amedee, "Microgliaderived purines modulate mossy fibre synaptic transmission and plasticity through P2X4 and A1 receptors," The European Journal of Neuroscience, vol. 43, no. 10, pp. 13661378, 2016.

[25] A. Araque, V. Parpura, R. P. Sanzgiri, and P. G. Haydon, "Tripartite synapses: glia, the unacknowledged partner," Trends in Neurosciences, vol. 22, no. 5, pp. 208-215, 1999.

[26] A. Araque, G. Carmignoto, P. G. Haydon, S. H. Oliet, R. Robitaille, and A. Volterra, "Gliotransmitters travel in time and space," Neuron, vol. 81, no. 4, pp. 728-739, 2014.

[27] N. B. Hamilton and D. Attwell, "Do astrocytes really exocytose neurotransmitters?" Nature Reviews Neuroscience, vol. 11, no. 4, pp. 227-238, 2010.

[28] U. Lalo, O. Palygin, S. Rasooli-Nejad, J. Andrew, P. G. Haydon, and Y. Pankratov, "Exocytosis of ATP from astrocytes modulates phasic and tonic inhibition in the neocortex," PLoS Biology, vol. 12, no. 1, article e1001747, 2014.

[29] Z. Zhang, G. Chen, W. Zhou et al., "Regulated ATP release from astrocytes through lysosome exocytosis," Nature Cell Biology, vol. 9, no. 8, pp. 945-953, 2007.

[30] G. R. Gordon, D. V. Baimoukhametova, S. A. Hewitt, W. R. Rajapaksha, T. E. Fisher, and J. S. Bains, "Norepinephrine triggers release of glial ATP to increase postsynaptic efficacy," Nature Neuroscience, vol. 8, no. 8, pp. 1078-1086, 2005.

[31] G. R. Gordon, K. J. Iremonger, S. Kantevari, G. C. EllisDavies, B. A. MacVicar, and J. S. Bains, "Astrocyte-mediated distributed plasticity at hypothalamic glutamate synapses," Neuron, vol. 64, no. 3, pp. 391-403, 2009.

[32] U. Lalo, O. Palygin, A. Verkhratsky, S. G. Grant, and Y. Pankratov, "ATP from synaptic terminals and astrocytes regulates NMDA receptors and synaptic plasticity through PSD-95 multi-protein complex," Scientific Reports, vol. 6, article 33609, 2016.

[33] S. Rasooli-Nejad, O. Palygin, U. Lalo, and Y. Pankratov, "Cannabinoid receptors contribute to astroglial $\mathrm{Ca}(2)(+)$-signalling and control of synaptic plasticity in the neocortex," Philosophical Transactions of the Royal Society of London Series B, Biological Sciences, vol. 369, no. 1654, article 20140077, 2014.

[34] S. A. Sloan and B. A. Barres, "Looks can be deceiving: reconsidering the evidence for gliotransmission," Neuron, vol. 84, no. 6, pp. 1112-1115, 2014.

[35] S. Sultan, L. Li, J. Moss et al., "Synaptic integration of adultborn hippocampal neurons is locally controlled by astrocytes," Neuron, vol. 88, no. 5, pp. 957-972, 2015. 
[36] T. Papouin, J. M. Dunphy, M. Tolman, K. T. Dineley, and P. G. Haydon, "Septal cholinergic neuromodulation tunes the astrocyte-dependent gating of hippocampal NMDA receptors to wakefulness," Neuron, vol. 94, no. 4, pp. 840-854, 2017, e7.

[37] J. M. Garre, M. A. Retamal, P. Cassina et al., "FGF-1 induces ATP release from spinal astrocytes in culture and opens pannexin and connexin hemichannels," Proceedings of the National Academy of Sciences of the United States of America, vol. 107, no. 52, pp. 22659-22664, 2010.

[38] C. Volonte, S. Amadio, F. Cavaliere, N. D'Ambrosi, F. Vacca, and G. Bernardi, "Extracellular ATP and neurodegeneration," Current Drug Targets CNS and Neurological Disorders, vol. 2, no. 6, pp. 403-412, 2003.

[39] U. Lalo, S. Rasooli-Nejad, and Y. Pankratov, "Exocytosis of gliotransmitters from cortical astrocytes: implications for synaptic plasticity and aging," Biochemical Society Transactions, vol. 42, no. 5, pp. 1275-1281, 2014.

[40] J. T. Pougnet, E. Toulme, A. Martinez, D. Choquet, E. Hosy, and E. Boue-Grabot, "ATP P2X receptors downregulate AMPA receptor trafficking and postsynaptic efficacy in hippocampal neurons," Neuron, vol. 83, no. 2, pp. 417-430, 2014.

[41] F. A. Edwards, A. J. Gibb, and D. Colquhoun, "ATP receptormediated synaptic currents in the central nervous system," Nature, vol. 359, no. 6391, pp. 144-147, 1992.

[42] F. A. Edwards, S. J. Robertson, and A. J. Gibb, "Properties of ATP receptor-mediated synaptic transmission in the rat medial habenula," Neuropharmacology, vol. 36, no. 9, pp. 1253-1268, 1997.

[43] Y. H. Jo and R. Schlichter, "Synaptic corelease of ATP and GABA in cultured spinal neurons," Nature Neuroscience, vol. 2, no. 3, pp. 241-245, 1999.

[44] Y. Pankratov, U. Lalo, O. Krishtal, and A. Verkhratsky, "Ionotropic P2X purinoreceptors mediate synaptic transmission in rat pyramidal neurones of layer II/III of somatosensory cortex," The Journal of Physiology, vol. 542, Part 2, pp. 529-536, 2002.

[45] Y. Pankratov, U. Lalo, A. Verkhratsky, and R. A. North, "Quantal release of ATP in mouse cortex," The Journal of General Physiology, vol. 129, no. 3, pp. 257-265, 2007.

[46] Y. V. Pankratov, U. V. Lalo, and O. A. Krishtal, "Role for P2X receptors in long-term potentiation," The Journal of Neuroscience, vol. 22, no. 19, pp. 8363-8369, 2002.

[47] Y.H. Jo and L. W. Role, "Coordinate release of ATP and GABA at in vitro synapses of lateral hypothalamic neurons," The Journal of Neuroscience, vol. 22, no. 12, pp. 4794-4804, 2002.

[48] E. Boue-Grabot, "Interactions between ligand-gated ion channels: a new regulation mechanism for fast synaptic signaling?" Amino Acid Receptor Research Nova Science Publishers, 2008.

[49] J. T. Pougnet, B. Compans, A. Martinez, D. Choquet, E. Hosy, and E. Boue-Grabot, "P2X-mediated AMPA receptor internalization and synaptic depression is controlled by two CaMKII phosphorylation sites on GluA1 in hippocampal neurons," Scientific Reports, vol. 6, article 31836, 2016.

[50] R. Gomez-Villafuertes, J. Gualix, and M. T. Miras-Portugal, "Single GABAergic synaptic terminals from rat midbrain exhibit functional P2X and dinucleotide receptors, able to induce GABA secretion," Journal of Neurochemistry, vol. 77, no. 1, pp. 84-93, 2001.
[51] J. G. Gu and A. B. MacDermott, "Activation of ATP P2X receptors elicits glutamate release from sensory neuron synapses," Nature, vol. 389, no. 6652, pp. 749-753, 1997.

[52] R. A. Cunha and J. A. Ribeiro, "ATP as a presynaptic modulator," Life Sciences, vol. 68, no. 2, pp. 119-137, 2000.

[53] M. Mori, C. Heuss, B. H. Gahwiler, and U. Gerber, "Fast synaptic transmission mediated by $\mathrm{P} 2 \mathrm{X}$ receptors in CA3 pyramidal cells of rat hippocampal slice cultures," The Journal of Physiology, vol. 535, Part 1, pp. 115-123, 2001.

[54] G. H. Hansen, E. Hosli, B. Belhage, A. Schousboe, and L. Hosli, "Light and electron microscopic localization of GABAA-receptors on cultured cerebellar granule cells and astrocytes using immunohistochemical techniques," Neurochemical Research, vol. 16, no. 3, pp. 341-346, 1991.

[55] A. Baude, Z. Nusser, E. Molnar, R. A. McIlhinney, and P. Somogyi, "High-resolution immunogold localization of AMPA type glutamate receptor subunits at synaptic and non-synaptic sites in rat hippocampus," Neuroscience, vol. 69, no. 4, pp. 1031-1055, 1995.

[56] A. Triller and D. Choquet, "Surface trafficking of receptors between synaptic and extrasynaptic membranes: and yet they do move!," Trends in Neurosciences, vol. 28, no. 3, pp. 133139, 2005.

[57] E. Toulme and B. S. Khakh, "Imaging P2X4 receptor lateral mobility in microglia: regulation by calcium and p38 MAPK," The Journal of Biological Chemistry, vol. 287, no. 18, pp. 14734-14748, 2012.

[58] E. Richler, E. Shigetomi, and B. S. Khakh, "Neuronal P2X2 receptors are mobile ATP sensors that explore the plasma membrane when activated," The Journal of Neuroscience, vol. 31, no. 46, pp. 16716-16730, 2011.

[59] L. K. Bobanovic, S. J. Royle, and R. D. Murrell-Lagnado, "P2X receptor trafficking in neurons is subunit specific," The Journal of Neuroscience, vol. 22, no. 12, pp. 4814-4824, 2002.

[60] Y. H. Jo, E. Donier, A. Martinez, M. Garret, E. Toulme, and E. Boue-Grabot, "Cross-talk between P2X4 and gammaaminobutyric acid, type A receptors determines synaptic efficacy at a central synapse," The Journal of Biological Chemistry, vol. 286, no. 22, pp. 19993-20004, 2011.

[61] S. J. Royle, L. K. Bobanovic, and R. D. Murrell-Lagnado, "Identification of a non-canonical tyrosine-based endocytic motif in an ionotropic receptor," The Journal of Biological Chemistry, vol. 277, no. 38, pp. 35378-35385, 2002.

[62] S. J. Royle, O. S. Qureshi, L. K. Bobanovic, P. R. Evans, D. J. Owen, and R. D. Murrell-Lagnado, "Non-canonical YXXGPhi endocytic motifs: recognition by AP2 and preferential utilization in P2X4 receptors," Journal of Cell Science, vol. 118, Part 14, pp. 3073-3080, 2005.

[63] E. Toulme, A. Garcia, D. Samways, T. M. Egan, M. J. Carson, and B. S. Khakh, "P2X4 receptors in activated C8-B4 cells of cerebellar microglial origin," The Journal of General Physiology, vol. 135, no. 4, pp. 333-353, 2010.

[64] L. E. Robinson and R. D. Murrell-Lagnado, "The trafficking and targeting of P2X receptors," Frontiers in Cellular Neuroscience, vol. 7, p. 233, 2013.

[65] R. Varma, Y. Chai, J. Troncoso et al., "Amyloid-beta induces a caspase-mediated cleavage of $\mathrm{P} 2 \mathrm{X} 4$ to promote purinotoxicity," Neuromolecular Medicine, vol. 11, no. 2, pp. 63-75, 2009.

[66] A. Casanovas, S. Hernandez, O. Tarabal, J. Rossello, and J. E. Esquerda, "Strong $\mathrm{P} 2 \mathrm{X} 4$ purinergic receptor-like 
immunoreactivity is selectively associated with degenerating neurons in transgenic rodent models of amyotrophic lateral sclerosis," The Journal of Comparative Neurology, vol. 506, no. 1, pp. 75-92, 2008.

[67] S. Hernandez, A. Casanovas, L. Piedrafita, O. Tarabal, and J. E. Esquerda, "Neurotoxic species of misfolded SOD1G93A recognized by antibodies against the $\mathrm{P} 2 \mathrm{X} 4$ subunit of the ATP receptor accumulate in damaged neurons of transgenic animal models of amyotrophic lateral sclerosis," Journal of Neuropathology and Experimental Neurology, vol. 69, no. 2, pp. 176-187, 2010.

[68] H. W. Kessels and R. Malinow, "Synaptic AMPA receptor plasticity and behavior," Neuron, vol. 61, no. 3, pp. 340350, 2009.

[69] R. Malinow and R. C. Malenka, "AMPA receptor trafficking and synaptic plasticity," Annual Review of Neuroscience, vol. 25, pp. 103-126, 2002.

[70] M. Heine, L. Groc, R. Frischknecht et al., "Surface mobility of postsynaptic AMPARs tunes synaptic transmission," Science, vol. 320, no. 5873, pp. 201-205, 2008.

[71] S. F. Traynelis, L. P. Wollmuth, C. J. McBain et al., "Glutamate receptor ion channels: structure, regulation, and function," Pharmacological Reviews, vol. 62, no. 3, pp. 405-496, 2010.

[72] R. A. Nicoll, S. Tomita, and D. S. Bredt, "Auxiliary subunits assist AMPA-type glutamate receptors," Science, vol. 311, no. 5765, pp. 1253-1256, 2006.

[73] V. Anggono and R. L. Huganir, "Regulation of AMPA receptor trafficking and synaptic plasticity," Current Opinion in Neurobiology, vol. 22, no. 3, pp. 461-469, 2012.

[74] V. Compan, L. Ulmann, O. Stelmashenko, J. Chemin, S. Chaumont, and F. Rassendren, "P2X2 and P2X5 subunits define a new heteromeric receptor with P2X7-like properties," The Journal of Neuroscience, vol. 32, no. 12, pp. 42844296, 2012.

[75] M. Paukert, A. Agarwal, J. Cha, V. A. Doze, J. U. Kang, and D. E. Bergles, "Norepinephrine controls astroglial responsiveness to local circuit activity," Neuron, vol. 82, no. 6, pp. 1263-1270, 2014.

[76] F. Ding, J. O'Donnell, A. S. Thrane et al., " $\alpha 1$-Adrenergic receptors mediate coordinated $\mathrm{Ca}^{2+}$ signaling of cortical astrocytes in awake, behaving mice," Cell Calcium, vol. 54, no. 6, pp. 387-394, 2013.

[77] Y. Pankratov, U. Lalo, O. A. Krishtal, and A. Verkhratsky, "P2X receptors and synaptic plasticity," Neuroscience, vol. 158, no. 1, pp. 137-148, 2009.

[78] J. A. Sim, S. Chaumont, J. Jo et al., “Altered hippocampal synaptic potentiation in P2X4 knock-out mice," The Journal of Neuroscience, vol. 26, no. 35, pp. 9006-9009, 2006.

[79] S. D. Silberberg, M. Li, and K. J. Swartz, "Ivermectin interaction with transmembrane helices reveals widespread rearrangements during opening of P2X receptor channels," Neuron, vol. 54, no. 2, pp. 263-274, 2007.

[80] E. Toulme, F. Soto, M. Garret, and E. Boue-Grabot, "Functional properties of internalization-deficient $\mathrm{P} 2 \mathrm{X} 4$ receptors reveal a novel mechanism of ligand-gated channel facilitation by ivermectin," Molecular Pharmacology, vol. 69, no. 2, pp. 576-587, 2006.

[81] A. W. Baxter, S. J. Choi, J. A. Sim, and R. A. North, "Role of $\mathrm{P} 2 \mathrm{X} 4$ receptors in synaptic strengthening in mouse CA1 hippocampal neurons," The European Journal of Neuroscience, vol. 34, no. 2, pp. 213-220, 2011.
[82] M. Migaud, P. Charlesworth, M. Dempster et al., "Enhanced long-term potentiation and impaired learning in mice with mutant postsynaptic density-95 protein," Nature, vol. 396, no. 6710, pp. 433-439, 1998.

[83] J. Nithianantharajah, N. H. Komiyama, A. McKechanie et al., "Synaptic scaffold evolution generated components of vertebrate cognitive complexity," Nature Neuroscience, vol. 16, no. 1, pp. 16-24, 2013.

[84] L. Atkinson, T. F. Batten, and J. Deuchars, "P2X(2) receptor immunoreactivity in the dorsal vagal complex and area postrema of the rat," Neuroscience, vol. 99, no. 4, pp. 683-696, 2000.

[85] M. J. Neal, J. R. Cunningham, and Z. Dent, "Modulation of extracellular GABA levels in the retina by activation of glial P2X-purinoceptors," British Journal of Pharmacology, vol. 124, no. 2, pp. 317-322, 1998.

[86] Y. H. Jo and E. Boue-Grabot, "Interplay between ionotropic receptors modulates inhibitory synaptic strength," Communicative \& Integrative Biology, vol. 4, no. 6, pp. 706-709, 2011.

[87] B. S. Khakh, J. A. Fisher, R. Nashmi, D. N. Bowser, and H. A. Lester, "An angstrom scale interaction between plasma membrane ATP-gated P2X2 and alpha4beta2 nicotinic channels measured with fluorescence resonance energy transfer and total internal reflection fluorescence microscopy," The Journal of Neuroscience, vol. 25, no. 29, pp. 6911-6920, 2005.

[88] B. S. Khakh, X. Zhou, J. Sydes, J. J. Galligan, and H. A. Lester, "State-dependent cross-inhibition between transmitter-gated cation channels," Nature, vol. 406, no. 6794, pp. 405-410, 2000.

[89] E. Boue-Grabot, C. Barajas-Lopez, Y. Chakfe et al., "Intracellular cross talk and physical interaction between two classes of neurotransmitter-gated channels," The Journal of Neuroscience, vol. 23, no. 4, pp. 1246-1253, 2003.

[90] E. Boue-Grabot, M. B. Emerit, E. Toulme, P. Seguela, and M. Garret, "Cross-talk and co-trafficking between rhol/GABA receptors and ATP-gated channels," The Journal of Biological Chemistry, vol. 279, no. 8, pp. 6967-6975, 2004.

[91] E. Boue-Grabot, E. Toulme, M. B. Emerit, and M. Garret, "Subunit-specific coupling between gamma-aminobutyric acid type A and P2X2 receptor channels," The Journal of Biological Chemistry, vol. 279, no. 50, pp. 52517-52525, 2004.

[92] E. Toulme, D. Blais, C. Leger et al., "An intracellular motif of $\mathrm{P} 2 \mathrm{X}(3)$ receptors is required for functional cross-talk with GABA(A) receptors in nociceptive DRG neurons," Journal of Neurochemistry, vol. 102, no. 4, pp. 1357-1368, 2007.

[93] M. B. Emerit, C. Baranowski, J. Diaz et al., "A new mechanism of receptor targeting by interaction between two classes of ligand-gated ion channels," The Journal of Neuroscience, vol. 36, no. 5, pp. 1456-1470, 2016.

[94] Y. Pankratov and U. Lalo, "Role for astroglial alpha1adrenoreceptors in gliotransmission and control of synaptic plasticity in the neocortex," Frontiers in Cellular Neuroscience, vol. 9, p. 230, 2015.

[95] S. Viana da Silva, M. G. Haberl, P. Zhang et al., "Early synaptic deficits in the APP/PS1 mouse model of Alzheimer's disease involve neuronal adenosine A2A receptors," Nature Communications, vol. 7, article 11915, 2016.

[96] S. R. Hulme, O. D. Jones, C. R. Raymond, P. Sah, and W. C. Abraham, "Mechanisms of heterosynaptic metaplasticity," Philosophical Transactions of the Royal Society of London 
Series B, Biological Sciences, vol. 369, no. 1633, article 20130148, 2014.

[97] B. StrooperDe and E. Karran, "The cellular phase of Alzheimer's disease," Cell, vol. 164, no. 4, pp. 603-615, 2016.

[98] J. J. Rodriguez-Arellano, V. Parpura, R. Zorec, and A. Verkhratsky, "Astrocytes in physiological aging and Alzheimer's disease," Neuroscience, vol. 323, pp. 170-182, 2016.

[99] T. Keck, T. Toyoizumi, L. Chen et al., "Integrating Hebbian and homeostatic plasticity: the current state of the field and future research directions," Philosophical Transactions of the Royal Society of London Series B, Biological Sciences, vol. 372, no. 1715, 2017.

[100] T. Takeuchi, A. J. Duszkiewicz, and R. G. Morris, "The synaptic plasticity and memory hypothesis: encoding, storage and persistence," Philosophical Transactions of the Royal Society of London Series B, Biological Sciences, vol. 369, no. 1633, article 20130288, 2014.

[101] B. S. Khakh and K. D. McCarthy, "Astrocyte calcium signaling: from observations to functions and the challenges therein," Cold Spring Harbor Perspectives in Biology, vol. 7, no. 4, article a020404, 2015.

[102] A. Panatier, J. Vallee, M. Haber, K. K. Murai, J. C. Lacaille, and R. Robitaille, "Astrocytes are endogenous regulators of basal transmission at central synapses," Cell, vol. 146, no. 5, pp. 785-798, 2011.

[103] R. Martin, R. Bajo-Graneras, R. Moratalla, G. Perea, and A. Araque, "Circuit-specific signaling in astrocyte-neuron networks in basal ganglia pathways," Science, vol. 349, no. 6249 , pp. 730-734, 2015.

[104] M. P. Kummer, T. Hammerschmidt, A. Martinez et al., "Ear2 deletion causes early memory and learning deficits in APP/ PS1 mice," The Journal of Neuroscience, vol. 34, no. 26, pp. 8845-8854, 2014.

[105] J. Nithianantharajah and A. J. Hannan, “The neurobiology of brain and cognitive reserve: mental and physical activity as modulators of brain disorders," Progress in Neurobiology, vol. 89, no. 4, pp. 369-382, 2009. 

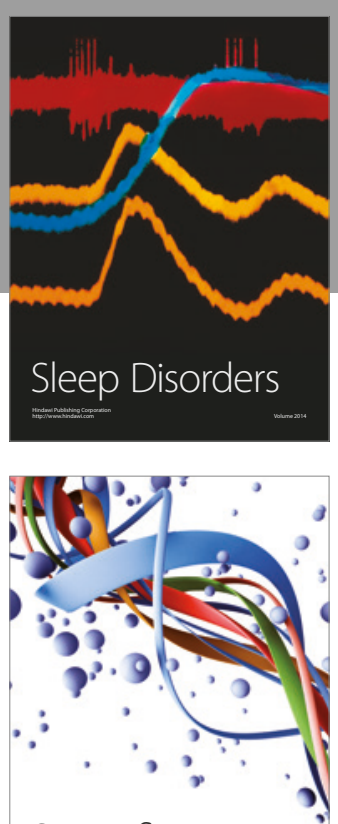

Scientifica
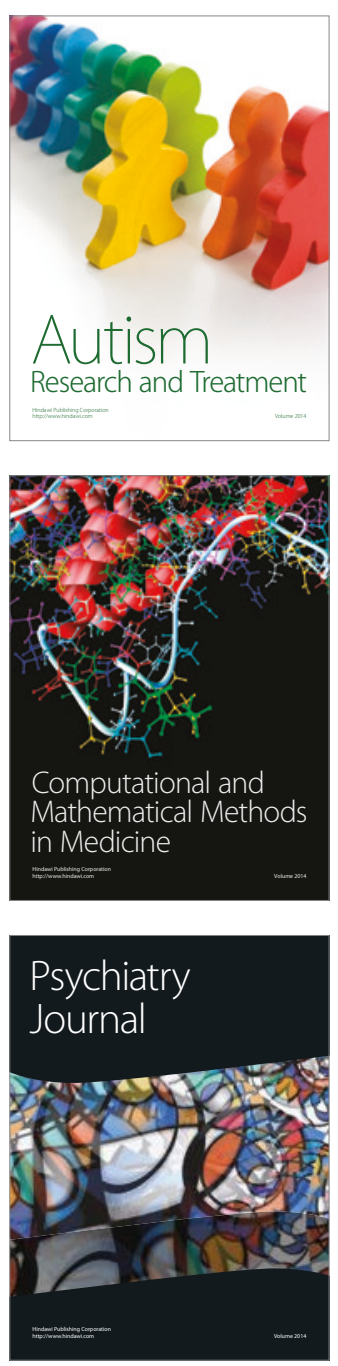
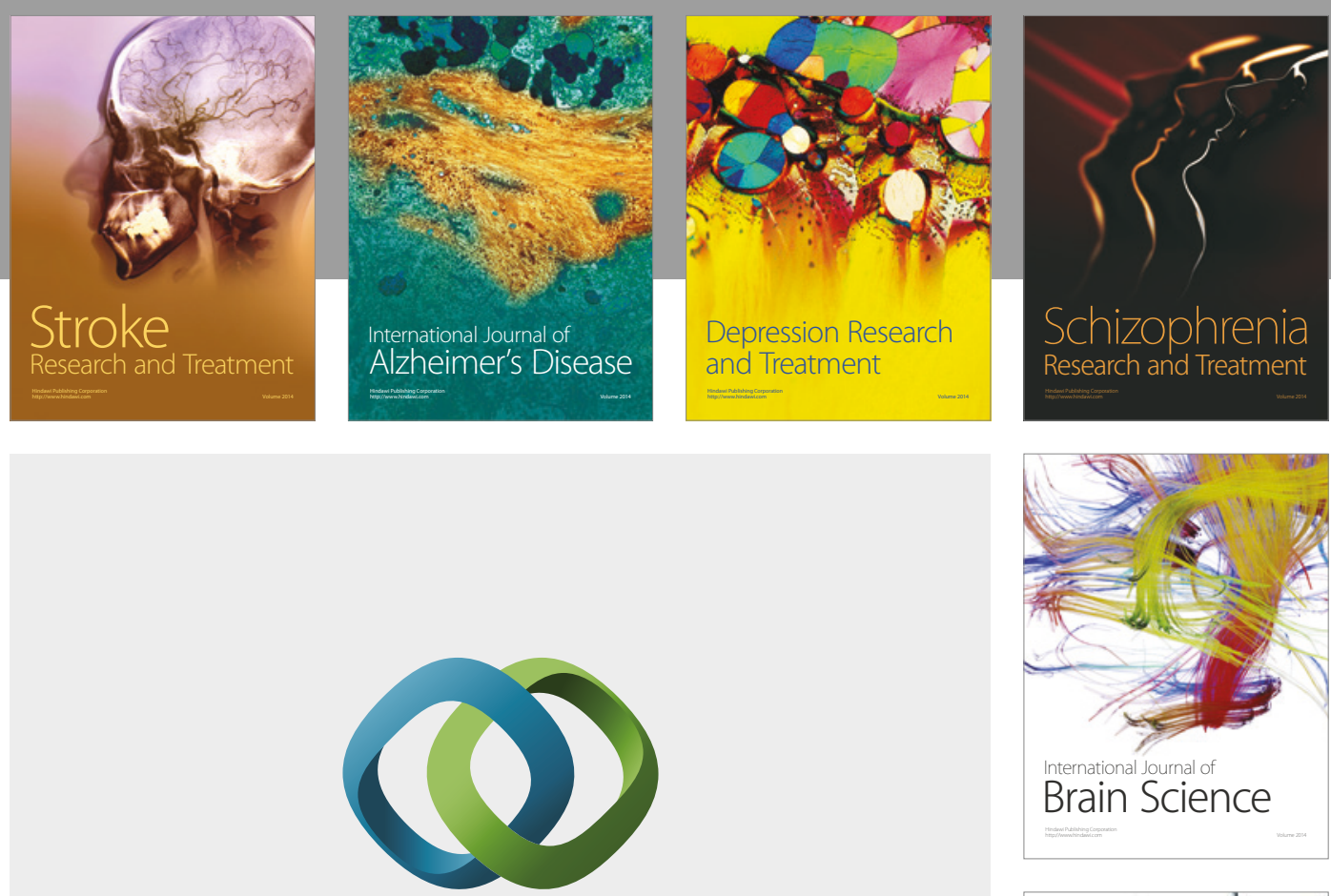

\section{Hindawi}

Submit your manuscripts at

https://www.hindawi.com
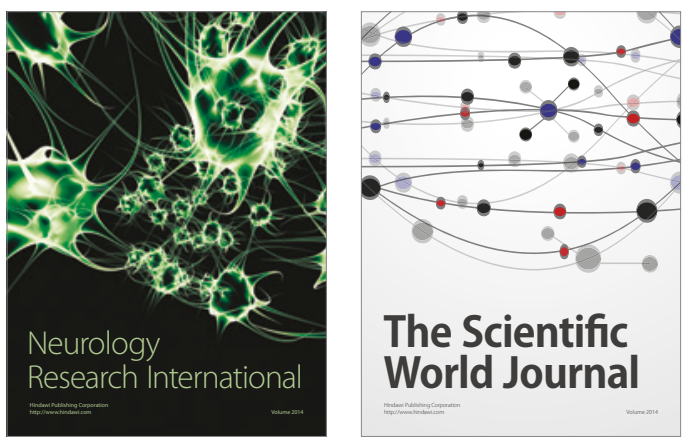

The Scientific World Journal

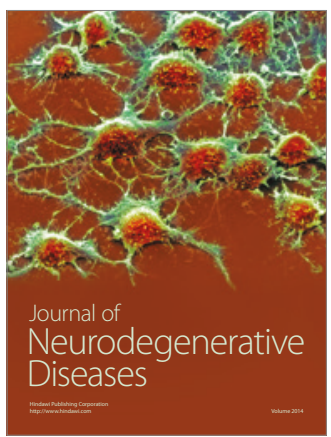

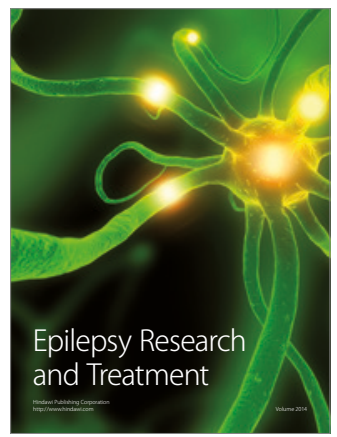

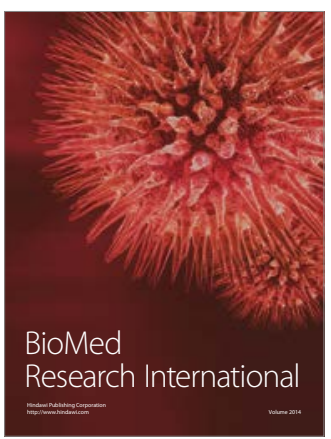

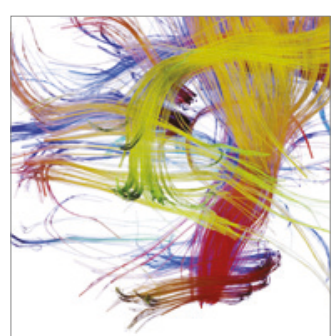

Brain Science

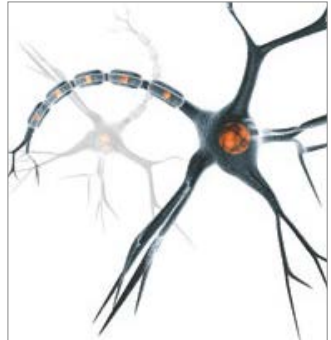

Neural Plasticity
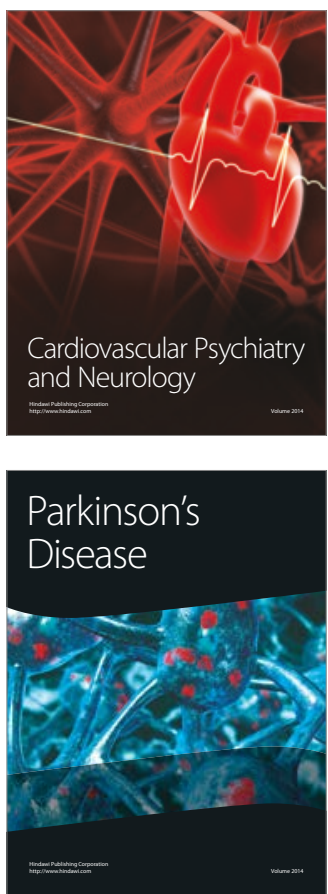